\title{
Selection of human p75NTR tag SNPs and its biological significance for clinical association studies
}

\author{
Yong-Tang Wang ${ }^{\mathrm{a},{ }^{*}}$, Xiu-Min Lu ${ }^{\mathrm{b}, *}$, Ya-Hai Shu ${ }^{\mathrm{b}}$, Lan Xiao ${ }^{\mathrm{b}}$ and Kai-Ting Chen ${ }^{\mathrm{b}}$ \\ ${ }^{a}$ State Key Laboratory of Trauma, Burns and Combined Injury, Institute of Surgery Research, Daping \\ Hospital, Third Military Medical University, Chongqing 400042, China \\ ${ }^{b}$ College of Pharmacy and Bioengineering, Chongqing University of Technology, Chongqing 400054, \\ China
}

\begin{abstract}
To select tag single nucleotide polymorphisms (SNPs) within and around human p75 neurotrophin receptor (p75NTR) gene in Chinese Han population, the sequence involving p75NTR gene as well as the upstream and downstream of the gene was identified according to the data from National Center for Biotechnology Information (NCBI) GenBank database, and the SNP genotype data involving 63 SNPs in the regions were obtained from Chinese Han Beijing (CHB) population of HapMap database. Then, Haploview (version 4.2) was used to calculate linkage disequilibrium (LD) statistics for the selected 32 common SNPs with a minor allele frequence (MAF) more than 0.05. Haplotype blocks were constructed throughout the p75NTR gene according to the upper and the lower 95\% confidence bound of D' value, and the tag SNPs were selected based on the $\mathrm{r}^{2}$ and LOD values between SNPs as well as the results of bioinformatics analysis. The results indicated that five haplotype blocks were constructed within and around p75NTR gene and 12 tag SNPs including rs2537710, rs603769, rs614455, rs2537706, rs534561, rs2072445, rs2072446, rs7219709, rs734194, rs741071, rs741073 and rs2671641 were selected to represent the other 51 SNPs in p75NTR gene. Therefore, the 12 selected SNPs may act as tag SNPs for the entire p75NTR gene in Chinese Han population, which will provide an effective way to select tag SNPs in a whole gene, and its biological significance is to further guide the clinical association studies between the candidate gene and disease susceptibility.
\end{abstract}

Keywords: p75NTR, haplotype block, tag SNP, haplotype, bioinformatics analysis

\section{Introduction}

As the first cloned low-affinity nerve growth factor receptor (NGFR), p75NTR can bind to all neurotrophic factors, mediating neuronal survival, growth and apoptosis, as well as regulation of synaptic plasticity. Besides, as a co-receptor for Nogo-66 receptor (Nogo-66 receptor, NgR), p75NTR is involved in the inhibition of neurite growth in the injured central nervous system (CNS) $[1,2]$. Recent studies showed that the expression of p75NTR in prefrontal cortex and hippocampus in suicide sub-

\footnotetext{
${ }^{*}$ Corresponding authors: Yong-Tang Wang, Institute of Surgery Research, Daping Hospital, Third Military Medical University, 10 Changjiang Sub-Road, Chongqing 400042, China. Tel.: +86 023 68757435; E-mail: wangytlu@163.com.

Xiu-Min Lu, College of Pharmacy and Bioengineering, Chongqing University of Technology, 69 Hongguang Road, Chongqing 400054, China. Tel.: +86 023 62563190; E-mail: luxm@cqut.edu.cn.
} 
jects with depression or other mental disorders is significantly higher than that in the normal subjects [3]. The results suggest that p75NTR plays an important role in the pathogenesis of mental disorders such as depression, posttraumatic stress disorder (PTSD) and Alzheimer's disease (AD), and may be a novel therapeutic target for neuropsychiatric diseases [1-3]. Growing evidences indicate that p75NTR may be involved in the occurrence of depression and its comorbid, such as schizophrenia and PTSD [4-8]. Therefore, p75NTR can be used as an important candidate gene for the study of genetic susceptibility to neuropsychiatric diseases. Recently, many clinical association studies between p75NTR gene polymorphism and susceptibility of mental disorders, including depression and suicidal tendency have been carried out. However, only a few p75NTR SNPs have been involved in previous studies [911], and little is known about the global biological significance of the SNPs within the entire p75NTR gene. To comprehensively assess the potential biologic significance of all known genetic variants within the entire p75NTR gene, a SNP haplotype tagging approach as well as bioinformatics analysis is used to select representative tag SNPs. It is thus possible that the study may provide an effective way to investigate the biological significance of all the SNPs within a whole gene, and further guide the clinical association studies between the candidate gene and disease susceptibility.

\section{Materials and methods}

\subsection{Selection of SNPs within and around 75 NTR gene}

The sequence within and around human p75NTR gene was identified according to the data from NCBI (www.ncbi.nlm.nih.gov) GenBank database (updated on Jun. 3, 2014). Then, all the SNPs within the whole p75NTR gene in 139 healthy CHB population were downloaded from the latest version of HapMap (www.hapmap.org) SNP database (HapMap Data Rel 28 phase II+III, August10, on NCBI B36 assembly, dbSNP b126, NGFR chr17: 44917666..44957360).

\subsection{Construction of haplotype blocks}

To select tag SNPs from the genetic variation data for the entire p75NTR gene, the common SNPs $(\mathrm{MAF}>0.05)$ were first selected, and haplotype blocks were constructed throughout the whole p75NTR gene, using Haploview, version 4.2 (Broad Institute of MIT and Harvard, Cambridge, Mass), a software package that provides computation of linkage disequilibrium (LD) statistics and population haplotype patterns from genotype data. Haplotype blocks represent regions with little evidence of historical recombination between common SNPs. The history of recombination between a pair of SNPs can be estimated with the use of the normalized measure of allelic association D' (value of D prime between the two loci) [12]. The criterion for SNPs selected to construct a haplotype block is that all SNPs in one region must be in strong LD with $\mathrm{D}^{\prime}$ greater than 0.98 for the upper $95 \%$ confidence bound $\left(\mathrm{C}_{\mathrm{U}}\right)$ and greater than 0.7 for the lower bound $\left(\mathrm{C}_{\mathrm{L}}\right)[13]$.

\subsection{Selection of tag SNPS}

A maximally informative haplotype tag SNPs (htSNPs) was then selected from each block by using software Tagger program (http://www.broad.mit.edu/mpg/haploview, updated on Sep. 15, 2009) [14]. This algorithm selects a subset of variants that capture all known common genetic variations in a gene, based on an LD threshold of $r^{2} \geq 0.8$ [15]. The inverse of $r^{2}$ represents the ratio of sample size needed 
to detect an indirect association with an unassayed SNP to direct association at the same power. Apart from htSNPs, some SNPs out of the blocks also should be selected based on an LD threshold of $\mathrm{r}^{2} \geq 0.8$, and others out of the blocks must be selected because they could not represent each other.

To further select the more representative tag SNPs with possible functional significance, whether the above selected tag SNPs are located in the repeat sequence of p75NTR gene was analyzed using online software (http://www.repeatmasker.org/cgi-bin/WEBRepeatMasker, updated on Jan. 31, 2014), and the SNPs from the 5'-flanking region and 3'UTR of the p75NTR gene were also analyzed using online software (http://www.genome.jp/tools/motif/, updated on Aug. 10, 1998) to inspect potential transcription factor binding sites. The SNPs in the repeat sequence should not be selected, while the SNPs influencing the transcription factor sites must be selected.

Table 1

Distribution of the common p75NTR gene SNPs in Chinese Han population

\begin{tabular}{|c|c|c|c|c|c|}
\hline SNP ID & Accession number & Location on the gene & Alleles $(\mathrm{A}: \mathrm{B})^{*}$ & MAF\# & Region \\
\hline 4 & rs585916 & -8907 & $\mathrm{C}: \mathrm{T}$ & 0.1 & 5'-flanking \\
\hline 5 & rs 1035050 & -8768 & $\mathrm{C}: \mathrm{T}$ & 0.198 & 5'-flanking \\
\hline 7 & rs2671687 & -7942 & $\mathrm{C}: \mathrm{T}$ & 0.209 & 5'-flanking \\
\hline 9 & rs2671686 & -7535 & $\mathrm{~T}: \mathrm{G}$ & 0.057 & 5'-flanking \\
\hline 13 & rs 2537710 & -6194 & $\mathrm{~A}: \mathrm{C}$ & 0.105 & 5'-flanking \\
\hline 17 & rs603769 & -2695 & $\mathrm{~A}: \mathrm{G}$ & 0.209 & 5'-flanking \\
\hline 18 & rs 2584665 & -2598 & $\mathrm{~A}: \mathrm{C}$ & 0.14 & 5'-flanking \\
\hline 20 & rs575791 & 1976 & $\mathrm{~A}: \mathrm{G}$ & 0.2 & Intron 1 \\
\hline 21 & rs9908234 & 4569 & $\mathrm{~A}: \mathrm{G}$ & 0.267 & Intron 1 \\
\hline 22 & rs600120 & 4705 & $\mathrm{~A}: \mathrm{G}$ & 0.07 & Intron 1 \\
\hline 23 & rs565042 & 5060 & $\mathrm{~A}: \mathrm{G}$ & 0.07 & Intron 1 \\
\hline 24 & rs3785931 & 5339 & $\mathrm{C}: \mathrm{T}$ & 0.476 & Intron 1 \\
\hline 25 & rs614455 & 5659 & $\mathrm{~T}: \mathrm{C}$ & 0.07 & Intron 1 \\
\hline 26 & rs2072444 & 5956 & $\mathrm{~T}: \mathrm{C}$ & 0.453 & Intron 1 \\
\hline 27 & rs657770 & 8371 & $\mathrm{C}: \mathrm{A}$ & 0.07 & Intron 2 \\
\hline 29 & rs2537706 & 13409 & $\mathrm{G}: \mathrm{A}$ & 0.122 & Intron 3 \\
\hline 30 & rs534561 & 13433 & $C: G$ & 0.344 & Intron 3 \\
\hline 31 & rs11466148 & 13451 & $\mathrm{C}: \mathrm{T}$ & 0.134 & Intron 3 \\
\hline 33 & rs3785930 & 14143 & $\mathrm{G}: \mathrm{A}$ & 0.07 & Intron 3 \\
\hline 36 & rs2072445 & 14933 & $\mathrm{G}: \mathrm{T}$ & 0.07 & Intron 3 \\
\hline 37 & rs2072446 & 15040 & $\mathrm{C}: \mathrm{T}$ & 0.148 & Exon 4 \\
\hline 42 & rs11466162 & 18116 & $\mathrm{G}: \mathrm{A}$ & 0.159 & 3'UTR \\
\hline 43 & rs7219709 & 18422 & $\mathrm{C}: \mathrm{T}$ & 0.136 & 3'UTR \\
\hline 44 & rs 1804011 & 18491 & $\mathrm{C}: \mathrm{A}$ & 0.116 & 3'UTR \\
\hline 46 & rs7224806 & 18685 & $\mathrm{~T}: \mathrm{C}$ & 0.198 & 3'UTR \\
\hline 47 & rs734194 & 18830 & $\mathrm{~T}: \mathrm{G}$ & 0.198 & 3'UTR \\
\hline 48 & rs741071 & 18911 & $\mathrm{C}: \mathrm{T}$ & 0.453 & 3'UTR \\
\hline 49 & rs741072 & 18924 & $\mathrm{C}: \mathrm{T}$ & 0.43 & 3'UTR \\
\hline 50 & rs741073 & 19107 & $\mathrm{G}: \mathrm{A}$ & 0.238 & 3'UTR \\
\hline 58 & rs2671641 & 24948 & $\mathrm{G}: \mathrm{C}$ & 0.155 & 3'UTR \\
\hline 59 & rs2671642 & 25037 & $\mathrm{~A}: \mathrm{G}$ & 0.163 & 3'UTR \\
\hline 60 & rs10491195 & 25517 & $\mathrm{~A}: \mathrm{G}$ & 0.105 & 3'UTR \\
\hline
\end{tabular}




\section{Results}

\subsection{Selection of SNPs within and around $p 75 N T R$ gene}

The full sequence of p75NTR gene with $39.728 \mathrm{~kb}$ in length included $10 \mathrm{~kb}$ upstream of the transcription start site, a total of 6 exons, 5 introns and $10 \mathrm{~kb}$ downstream of the stop codon, which was located on chromosome 17, position 49485293 to 49525020 (GenBank: NC000017). Total 63 SNPs within the whole p75NTR gene were obtained from the HapMap project for 139 healthy CHB population. Analysis of the genetic variation data revealed that there were 32 common SNPs (MAF>0.05), mainly distributed in 5'-flanking, intron, exon and 3' untranslated region (UTR) (see Table 1).

\subsection{Construction of haplotype blocks}

Fifteen of the selected SNPs constructed five blocks according to the criterion to construct a haplotype block (see Table 1 and Figure 1): block 1: from -2695 to 1976, included 3 SNPs (rs603769, rs2584665 and rs575791), located in p75NTR gene from 5'-flanking to Intron 1; block 2: from 4705 to 8371 included 6 SNPs (rs600120, rs565042, rs3785931, rs614455, rs2072444 and rs657770), located in Intron 1; block 3: from 18685 to 18830 , included 2 SNPs (rs7224806 and rs734194), located in 3'UTR; block 4: from 18911 to 18924, included 2 SNPs (rs741071 and rs741072), located in 3'UTR and block 5: from 24948 to 25037, included two SNPs (rs2671641 and rs2671642), also located in 3 'UTR. The total length of five blocks is $8588 \mathrm{bp}, 21.62 \%$ of the length of the total studied sequence, containing a total of 15 SNPs, which is $46.88 \%$ of the selected 32 SNPs.

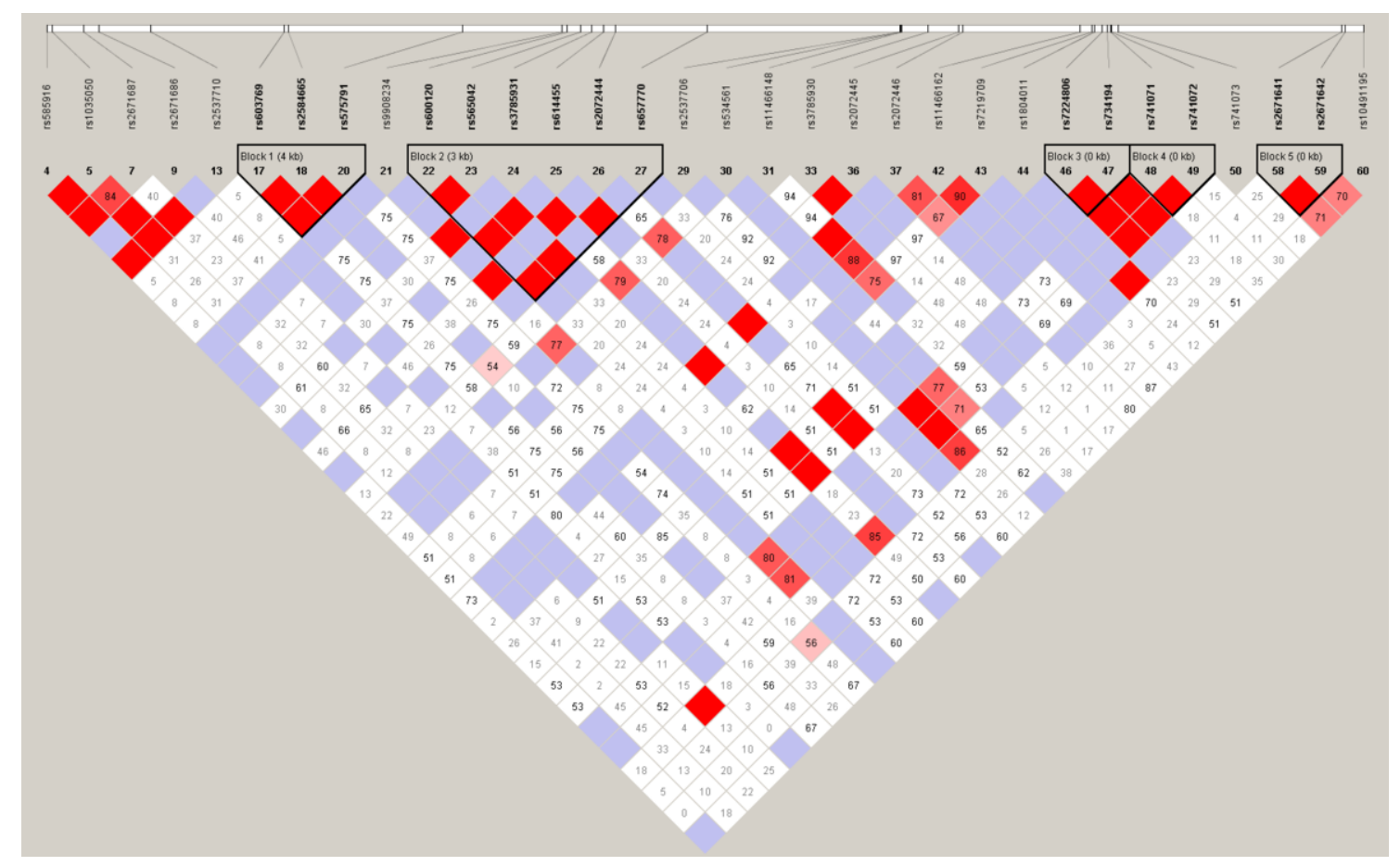

Fig. 1. Gene map and LD plot of common p75NTR gene SNPs in Chinese Han population: LD strength between SNPs, as indicated by the color scheme, was measured using a combination of the statistic $\mathrm{D}^{\prime}$ and the LOD score (dark shade, $\mathrm{D}^{\prime}=1$ and LOD score $\geq 3$; lighter shades, $\mathrm{D}^{\prime}<1$ and LOD score $<3$ ). Numbers in squares are $\mathrm{D}^{\prime}$ values. 


\subsection{Selection of tag SNPS}

Five SNPs, including rs603769, rs614455, rs734194, rs741071 and rs2671641, were separately selected as htSNPs from the 5 blocks, based on the analysis of tagging threshold of $\mathrm{r}^{2}$ of SNPs in each block (see Table 2). Some SNPs out of the blocks, such as rs2537710, rs2072445, rs2072446 and rs7219709, are also selected for tag SNPs according to the LD threshold of $r^{2} \geq 0.8$, other 9 SNPs out of the blocks, such as rs1035050, rs2671687, rs2671686, rs9908234, rs2537706, rs534561, rs1804011, rs741073 and rs10491195, are selected for tag SNPs because they could not represent other SNPs. However, the results of bioinformatics analysis indicated that the SNPs, such as rs1035050, rs2671687 and rs2671686 from the 5'-flanking region as well as rs1804011 and rs10491195 in 3'UTR, have no effects on the transcription factor sites. In addition, rs9908234 is located in the repeat sequence of p75NTR gene. As a result, among the 9 SNPs mentioned above, only rs2537706, rs534561 and rs741073 may have functional significance. Taken together, a total of 12 tag SNPs selected in present study can capture most of SNPs within the whole p75NTR gene and might represent the possible biological significance of the p75NTR genetic variation (see Table 3).

Table 2

The linkage disequilibrium, $\mathrm{r}^{2}$ value and LOD value between SNPs in the blocks of p75NTR gene

\begin{tabular}{|c|c|c|c|c|c|c|c|c|c|}
\hline Block & SNP & SNP & $\mathrm{D}^{\prime}$ & $\mathrm{C}_{\mathrm{L}} *$ & $\mathrm{C}_{\mathrm{U}} \#$ & $\mathrm{r}^{2}$ & LOD & Average $r^{2}$ & Average LOD \\
\hline \multirow[t]{4}{*}{1} & rs603769 & rs2584665 & 1 & 0.74 & 1 & 0.613 & 6.81 & 0.807 & 10.575 \\
\hline & & rs575791 & 1 & 0.89 & 1 & 1 & 14.34 & & \\
\hline & rs 2584665 & rs575791 & 1 & 0.74 & 1 & 0.613 & 6.81 & 0.613 & 6.81 \\
\hline & rs575791 & & & & & & & 0.807 & 10.575 \\
\hline \multirow[t]{16}{*}{2} & rs 600120 & rs565042 & 1 & 0.75 & 1 & 1 & 7.64 & 0.635 & 4.946 \\
\hline & & rs3785931 & 1 & 0.13 & 0.99 & 0.085 & 0.84 & & \\
\hline & & rs614455 & 1 & 0.75 & 1 & 1 & 7.64 & & \\
\hline & & rs2072444 & 1 & 0.15 & 0.99 & 0.09 & 0.97 & & \\
\hline & & rs 657770 & 1 & 0.75 & 1 & 1 & 7.64 & & \\
\hline & rs 565042 & rs3785931 & 1 & 0.13 & 0.99 & 0.085 & 0.84 & 0.635 & 4.946 \\
\hline & & rs614455 & 1 & 0.75 & 1 & 1 & 7.64 & & \\
\hline & & rs2072444 & 1 & 0.15 & 0.99 & 0.09 & 0.97 & & \\
\hline & & rs 657770 & 1 & 0.75 & 1 & 1 & 7.64 & & \\
\hline & rs3785931 & rs614455 & 1 & 0.13 & 0.99 & 0.085 & 0.84 & 0.259 & 3.92 \\
\hline & & rs2072444 & 1 & 0.9 & 1 & 0.953 & 16.24 & & \\
\hline & & rs657770 & 1 & 0.13 & 0.99 & 0.085 & 0.84 & & \\
\hline & rs614455 & rs2072444 & 1 & 0.15 & 0.99 & 0.09 & 0.97 & 0.635 & 4.946 \\
\hline & & rs657770 & 1 & 0.75 & 1 & 1 & 7.64 & & \\
\hline & rs 2072444 & rs657770 & 1 & 0.15 & 0.99 & 0.09 & 0.97 & 0.263 & 4.024 \\
\hline & rs 657770 & & & & & & & 0.635 & 4.946 \\
\hline \multirow[t]{2}{*}{3} & rs 7224806 & rs734194 & 1 & 0.89 & 1 & 1 & 14.05 & 1 & 14.05 \\
\hline & rs734194 & & & & & & & 1 & 14.05 \\
\hline \multirow[t]{2}{*}{4} & rs741071 & rs 741072 & 1 & 0.89 & 1 & 0.91 & 15.78 & 0.91 & 15.78 \\
\hline & rs741072 & & & & & & & 0.91 & 15.78 \\
\hline \multirow[t]{2}{*}{5} & rs 2671641 & rs2671642 & 1 & 0.82 & 1 & 0.915 & 10.54 & 0.915 & 10.54 \\
\hline & rs 2671642 & & & & & & & 0.915 & 10.54 \\
\hline
\end{tabular}

Note: *: $\mathrm{C}_{\mathrm{L}}$, the lower $95 \%$ confidence bound of the $\mathrm{D}^{\prime}$ value; $\#$ : $\mathrm{C}_{\mathrm{U}}$, the upper $95 \%$ confidence bound of the $\mathrm{D}^{\prime}$ value. 
Table 3

Tag SNPs of p75NTR gene

\begin{tabular}{|c|c|c|c|c|c|c|}
\hline Block & Tag SNP & Captured SNPs & Location on the gene & Variation $(\mathrm{A} / \mathrm{B})^{*}$ & MAF\# & Region \\
\hline & rs2537710 & rs585916, rs2537710 & -6194 & $\mathrm{~A} / \mathrm{C}$ & 0.105 & 5'-flanking \\
\hline 1 & rs603769 & $\begin{array}{l}\text { rs603769, rs2584665, } \\
\text { rs575791 }\end{array}$ & -2695 & $\mathrm{~A} / \mathrm{G}$ & 0.209 & 5'-flanking \\
\hline \multirow[t]{6}{*}{2} & rs614455 & $\begin{array}{l}\text { rs614455, } \\
\text { rs600120, } \\
\text { rs656565047, }\end{array}$ & 5659 & $\mathrm{~T} / \mathrm{C}$ & 0.07 & Intron 1 \\
\hline & rs2537706 & rs2537706 & 13409 & $\mathrm{G} / \mathrm{A}$ & 0.122 & Intron 3 \\
\hline & rs534561 & rs534561 & 13433 & $\mathrm{C} / \mathrm{G}$ & 0.344 & Intron 3 \\
\hline & rs2072445 & rs 2072445, rs 3785930 & 14933 & $\mathrm{G} / \mathrm{T}$ & 0.07 & Intron 3 \\
\hline & rs2072446 & rs11466148, rs2072446 & 15040 & $\mathrm{C} / \mathrm{T}$ & 0.148 & Exon 4 \\
\hline & rs7219709 & rs11466162, rs7219709 & 18422 & $\mathrm{C} / \mathrm{T}$ & 0.136 & 3'UTR \\
\hline 3 & rs734194 & rs 734194, rs 7224806 & 18830 & $\mathrm{~T} / \mathrm{G}$ & 0.198 & 3'UTR \\
\hline 4 & rs 741071 & rs 741071, rs 741072 & 18911 & $\mathrm{C} / \mathrm{T}$ & 0.453 & 3'UTR \\
\hline & rs 741073 & rs 741073 & 19107 & $\mathrm{G} / \mathrm{A}$ & 0.238 & 3'UTR \\
\hline 5 & rs2671641 & rs2671642, rs 2671641 & 24948 & $\mathrm{G} / \mathrm{C}$ & 0.155 & 3'UTR \\
\hline
\end{tabular}

Note: *: A, wild type allele; B, mutant type allele; \#: MAF, minor allele frequence.

\section{Discussion}

Compared with Trk receptor, p75NTR lacks enzyme activity and thus could not trigger a series of phosphorylation cascades by tyrosine kinase. However, as a low affinity neurotrophin receptor, p75NTR can bind to all neurotrophic factors, mediate neuronal survival and growth, induce neuronal apoptosis, and regulate synaptic plasticity. In addition, as a co-receptor for $\mathrm{NgR}$, it is involved in the inhibition of neurite growth in the injured CNS [1,2]. Studies showed that the expression of p75NTR in prefrontal cortex and hippocampus in suicide subjects with depression or other mental disorders is significantly up-regulated [3]. The results suggest that p75NTR plays a pivotal role in the pathogenesis of mental disorders such as depression, PTSD and AD, and may be a novel therapeutic target for neuropsychiatric diseases [1-3]. Indeed it has been shown that p75NTR, with neuronal cell apoptotic effects and regulation of synaptic plasticity, is involved in the occurrence of depression and its comorbid, such as schizophrenia and PTSD [4-8]. As a result, p75NTR can be used as an important candidate gene for the study of genetic susceptibility to neuropsychiatric diseases. Recently, many clinical association studies between p75NTR gene polymorphism and susceptibility of mental disorders, including depression and suicidal tendency, have been carried out. However, only a few p75NTR SNPs, such as rs575791, rs2537706, rs2072446, rs11466155 and rs734194, have been involved in previous studies [9-11], and little is known about the global biological significance of the genetic variants within the whole $\mathrm{p} 75 \mathrm{NTR}$ gene.

To address this problem, tag SNPs should be selected to represent the other SNPs in entire p75NTR gene. For this purpose, the sequence within and around human p75NTR gene is identified according to the data from NCBI GenBank database, and the SNP genotype data involving 63 SNPs of p75NTR gene are obtained from CHB population of HapMap database. Then, Haploview (version 4.2) is used to calculate LD statistics for the selected 32 common SNPs (MAF>0.05). Haplotype blocks are constructed throughout the p75NTR gene according to $\mathrm{C}_{\mathrm{U}}$ and $\mathrm{C}_{\mathrm{L}}$, and the tag SNPs are selected based on the $r^{2}$ and LOD values between SNPs as well as the results of bioinformatics analysis. The results indicate that five haplotype blocks are constructed and a total of 12 tag SNPs including rs2537710, 
rs603769, rs614455, rs2537706, rs534561, rs2072445, rs2072446, rs7219709, rs734194, rs741071, rs741073 and rs2671641 are selected to capture most of the genetic variation of the entire p75NTR gene and might represent the possible biological significance of the p75NTR genetic variation. Therefore, future research will be conducted to investigate the clinical association studies between the selected tag SNPs of p75NTR gene and the susceptibility of mental disorders such as depression, PTSD and AD. Taken together, the method to select tag SNPs in this study may provide an effective way to select tag SNPs in a whole gene, and its biological significance is to further guide the clinical association studies between the candidate gene and disease susceptibility.

\section{Acknowledgement}

This work was supported by the National Natural Science Foundation of China (81372040).

\section{References}

[1] T. Fujii and H. Kunugi, p75NTR as a therapeutic target for neuropsychiatric diseases, Curr. Mol. Pharmacol. 2 (2009), $70-76$.

[2] F. Zeng, J.J. Lu, X.F. Zhou and Y.J. Wang, Roles of p75NTR in the pathogenesis of Alzheimer's disease: A novel therapeutic target, Biochem. Pharmacol. 82 (2011), 1500-1509.

[3] Y. Dwivedi, H.S. Rizavi, H. Zhang, A.C. Mondal, R.C. Roberts, R.R. Conley, N. Ghanshyam and G.N. Pandey, Neurotrophin receptor activation and expression in human postmortem brain: Effect of suicide, Biol. Psychiat. 65 (2009), 319-328.

[4] X.M. Li, F. Han, D.J. Liu and Y.X. Shi, Single-prolonged stress induced mitochondrial-dependent apoptosis in hippocampus in the rat model of post-traumatic stress disorder, J. Chem. Neuroanat. 40 (2010), 248-255.

[5] B. Xiao, B. Yu, H.T. Wang, F. Han and Y.X. Shi, Single-prolonged stress induces apoptosis by activating Cytochrome $\mathrm{C} /$ Caspase-9 pathway in a rat model of post-traumatic stress disorder, Cell Mol. Neurobiol. 31 (2011), 37-43.

[6] J. Ding, F. Han and Y. Shi, Single-prolonged stress induces apoptosis in the amygdala in a rat model of post-traumatic stress disorder, J. Psychiatr. Res. 44 (2010), 48-55.

[7] N.H. Woo, H.K. Teng, C.J. Siao, C. Chiaruttini, P.T. Pang, T.A. Milner, B.L. Hempstead and B. Lu, Activation of p75NTR by proBDNF facilitates hippocampal long-term depression, Nat. Neurosci. 8 (2005), 1069-1077.

[8] K. Martinowich, R.J. Schloesser, Y. Lu, D.V. Jimenez, D. Paredes, J.S. Greene, N.H. Greig, H.K. Manji and B. Lu, Roles of p75NTR, Long-term depression, and cholinergic transmission in anxiety and acute stress coping, Biol. Psychiatry 71 (2012), 75-83.

[9] H. Kunugi, R. Hashimoto, M. Yoshida, M. Tatsumi and K. Kamijima, A missense polymorphism (S205L) of the lowaffinity neurotrophin receptor p75NTR gene is associated with depressive disorder and attempted suicide, Am. J. Med. Genet. Part B 129B (2004), 44-46.

[10] T. Fujii, N. Yamamoto, H. Hori, K. Hattori, D. Sasayama, T. Teraishi, M. Hashikura, M. Tatsumi, N. Okamoto, T. Higuchi and H. Kunugi, Support for association between the Ser205Leu polymorphism of p75NTR and major depressive disorder, J. Hum. Genet. 56 (2011), 806-809.

[11] Y.T.A. Gau, Y.J. Liou, Y.W.Y. Yu, T.J. Chen, M.W. Lin, S.J. Tsai and C.J. Hong, Evidence for association between genetic variants of p75 neurotrophin receptor (p75NTR) gene and antidepressant treatment response in Chinese major depressive disorder, Am. J. Med. Genet. Part B 147B (2008), 594-599.

[12] J.C. Barrett, B. Fry, J. Maller and M.J. Daly, Haploview: Analysis and visualization of LD and haplotype maps, Bioinformatics 21 (2005), 263-265.

[13] M.J. Daly, J.D. Rioux, S.F. Schaffner, T.J. Hudson and E.S. Lander, High-resolution haplotype structure in the human genome, Nat. Genet. 29 (2001), 229-232.

[14] Z. Xu, N.L. Kaplan and J.A. Taylor, TAGster: Efficient selection of LD tag SNPs in single or multiple populations, Bioinformatics 23 (2007), 3254-3255.

[15] C.S. Carlson, M.A. Eberle, M.J. Rieder, Q. Yi, L. Kruglyak and D.A. Nickerson, Selecting a maximally informative set of single nucleotide polymorphisms for association analyses using linkage disequilibrium, Am. J. Hum. Genet. 74 (2004), 106-120. 